\title{
WANDERING DOMAINS IN NON-ARCHIMEDEAN POLYNOMIAL DYNAMICS
}

\author{
ROBERT L. BENEDETTO
}

\begin{abstract}
We extend a recent result on the existence of wandering domains of polynomial functions defined over the $p$-adic field $\mathbb{C}_{p}$ to any algebraically closed complete non-archimedean field $\mathbb{C}_{K}$ with residue characteristic $p>0$. We also prove that polynomials with wandering domains form a dense subset of a certain one-dimensional family of degree $p+1$ polynomials in $\mathbb{C}_{K}[z]$.
\end{abstract}

Given a rational function $\phi \in K(z)$ with coefficients in a field $K$, one may consider the dynamical system given by the action of the iterates $\phi^{n}$ on $\mathbb{P}^{1}(K)=K \cup\{\infty\}$, for $n \geq 0$. Here, $\phi^{n}$ denotes the $n$-fold composition $\phi \circ \cdots \circ \phi$, so that $\phi^{0}$ is the identity function, $\phi^{1}=\phi, \phi^{2}=\phi \circ \phi$, and so on. The case of complex dynamics, when $K=\mathbb{C}$, has been studied intensively for several decades; see [1, 9, 15] for expositions. In particular, one may study the action of $\left\{\phi^{n}\right\}$ on the Fatou set $\mathcal{F}=\mathcal{F}_{\phi}$, to be defined below. It is well known that the connected components of $\mathcal{F}$ are mapped onto one another by $\phi$ and that, according to Sullivan's deep No Wandering Domains Theorem [20], every complex Fatou component is preperiodic under application of $\phi$.

It is also possible to define Fatou sets for other metric fields. The study of the resulting dynamics has seen growing interest in the past decade or two; see, for example, [2, 8, 11, 12, 14, 17. In this paper we will study wandering domains over certain non-archimedean fields, and we fix the following notation.

$\begin{array}{ll}\mathbb{C}_{K} & \text { a complete and algebraically closed non-archimedean field } \\ |\cdot| & \text { the absolute value on } \mathbb{C}_{K} \\ \hat{k} & \text { the residue field of } \mathbb{C}_{K} \\ p & \text { the residue characteristic char } \hat{k} \\ \mathbb{P}^{1}\left(\mathbb{C}_{K}\right) & \text { the projective line } \mathbb{C}_{K} \cup\{\infty\}\end{array}$

We will assume throughout that $p>0$; that is, that $\mathbb{C}_{K}$ has positive residue characteristic. Note that $0 \leq|p|<1$, when $p$ is viewed as an element of $\mathbb{C}_{K}$ under the unique nontrivial homomorphism of $\mathbb{Z}$ into $\mathbb{C}_{K}$. It is possible that char $\mathbb{C}_{K}=0$ with char $\hat{k}=p>0$; but if char $\mathbb{C}_{K}>0$, then char $\hat{k}=\operatorname{char} \mathbb{C}_{K}$.

Recall that "non-archimedean" means $\mathbb{C}_{K}$ satisfies the ultrametric triangle inequality

$$
|x+y| \leq \max \{|x|,|y|\} \quad \text { for all } \quad x, y \in \mathbb{C}_{K} .
$$

If $|x| \neq|y|$, it is immediate that $|x+y|=\max \{|x|,|y|\}$. Note that $|n| \leq 1$ for all $n \in \mathbb{Z}$. Recall also that the residue field $\hat{k}$ is defined to be $\mathcal{O}_{K} / \mathcal{M}_{K}$, where $\mathcal{O}_{K}$ is the ring $\left\{x \in \mathbb{C}_{K}:|x| \leq 1\right\}$ of integers in $\mathbb{C}_{K}$, and $\mathcal{M}_{K}$ is the maximal ideal of $\{x \in K:|x|<1\}$ of $\mathcal{O}_{K}$. It is easy to check that $\hat{k}$ is algebraically closed because $\mathbb{C}_{K}$ is.

Date: November 8, 2003; revised February 10, 2006.

2000 Mathematics Subject Classification. Primary: 12J25; Secondary: 37F99.

The author gratefully acknowledges the support of NSF grant DMS-0071541. 
The best known example of such a field is $\mathbb{C}_{p}$, constructed as follows. Given a prime integer $p \geq 2$, the (complete non-archimedean) field $\mathbb{Q}_{p}$ of $p$-adic rational numbers is the completion of the usual rational numbers $\mathbb{Q}$ with respect to the $p$-adic absolute value given by $\left|p^{e} r\right|=p^{-e}$, where $r$ is a rational number with numerator and denominator both prime to $p$. Its algebraic closure is $\overline{\mathbb{Q}}_{p}$, and the completion of $\overline{\mathbb{Q}}_{p}$ is $\mathbb{C}_{p}$; the absolute value extends uniquely to $\overline{\mathbb{Q}}_{p}$ and hence to $\mathbb{C}_{p}$. The residue field is $\hat{k}=\overline{\mathbb{F}}_{p}$, the algebraic closure of the field $\mathbb{F}_{p}$ of $p$ elements. Note that char $\mathbb{C}_{p}=\operatorname{char} \mathbb{Q}_{p}=0$, while char $\hat{k}=p>0$.

As another example, if $L$ is any abstract field, then $K=L((T))$, the field of formal Laurent series with coefficients in $L$, is a complete non-archimedean field. (We may define an absolute value $|\cdot|$ on $K$ by $|f|=2^{-n}$, where $n \in \mathbb{Z}$ is the smallest integer for which the $T^{n}$ term of the formal Laurent series $f$ has a nonzero coefficient.) Once again, the absolute value extends uniquely to an algebraic closure of $K$. If we denote by $\mathbb{C}_{K}$ the completion of an algebraic closure of $K$, then $\mathbb{C}_{K}$ is algebraically closed and complete; its residue field $\hat{k}$ is an algebraic closure of $L$. In this case, char $\mathbb{C}_{K}=\operatorname{char} \hat{k}=$ char $K=$ char $L$. We refer the reader to [13, 18, 19] for surveys of non-archimedean fields.

In complex dynamics, given a rational function $\phi \in \mathbb{C}(z)$ with complex coefficients, one considers the action of the iterates $\left\{\phi^{n}\right\}$ on the Riemann sphere $\mathbb{P}^{1}(\mathbb{C})$. The Fatou set $\mathcal{F}$ of $\phi$ is defined to be the the set of all points $x \in \mathbb{P}^{1}(\mathbb{C})$ such that the family $\left\{\phi^{n}: n \geq 0\right\}$ is equicontinuous at $x$, with respect to the spherical metric on $\mathbb{P}^{1}(\mathbb{C})$; the Julia set $\mathcal{J}$ is the complement $\mathbb{P}^{1}(\mathbb{C}) \backslash \mathcal{F}$. (Recall that a family $F$ of functions from a metric space $X$ to a metric space $Y$ is called equicontinuous at $x_{0} \in X$ if for every $\varepsilon>0$ there is a $\delta>0$ such that $d_{Y}\left(f(x), f\left(x_{0}\right)\right)<\varepsilon$ for all $f \in F$ and for all $x \in X$ satisfying $d_{X}\left(x, x_{0}\right)<\delta$. The key point is that $\varepsilon$ is chosen independent of $f$.) Intuitively, the Fatou set is the region of order, where the iterates $\left\{\phi^{n}\right\}$ are well behaved; the Julia set is the region of chaos, where a small error becomes huge after many iterations of $\phi$.

Alternately, by the Arzelà-Ascoli Theorem, one may define the Fatou set of a complex function to be the set of points where $\left\{\phi^{n}\right\}$ forms a normal family. However, because $\mathbb{C}_{K}$ is not locally compact, and therefore the Arzelà-Ascoli Theorem fails, non-archimedean Fatou sets are usually defined in terms of equicontinuity.

It is easy to verify that the Fatou set is open and that $\phi(\mathcal{F})=\mathcal{F}$; similarly, the Julia set is closed, and $\phi(\mathcal{J})=\mathcal{J}$. One may partition the (complex) Fatou set of $\phi \in \mathbb{C}(z)$ into connected components; the function $\phi$ then maps each component into (and in fact onto) a component. Thus, we may speak of fixed, periodic, and preperiodic components. A component which is not preperiodic (that is, a component with infinite forward orbit) is called a wandering domain of $\phi$. In 1985, Sullivan [20] proved, using quasiconformal conjugations, that a complex rational function $\phi \in \mathbb{C}(z)$ cannot have wandering domains.

A similar theory of components of the Fatou set exists for non-archimedean rational functions $\phi \in \mathbb{C}_{K}(z)$; see Section 1 below, where we give a precise characterization of such components for our setting. However, in spite of the existence of certain non-archimedean versions of Teichmüller spaces [16], there is no apparent analogue of actual quasiconformal maps suitable for use as conjugating functions in $\mathbb{C}_{K}(z)$. In particular, the author's constructions of wandering domains over any algebraically closed complete non-archimedean field (see [7] for residue characteristic zero, [5] for $\mathbb{C}_{p}$, and this paper for general fields of positive residue characteristic) suggest that Sullivan's proof has no analogue for $\mathbb{C}_{K}$, and that no appropriate versions of quasiconformal maps exist in that context. 
Nevertheless, the author showed in [3] that given some weak hypotheses on $\phi \in \mathbb{C}_{p}(z)$ (including the assumption that the coefficients of $\phi$ are algebraic over $\mathbb{Q}_{p}$ ), the Fatou set has no wandering domains. For more general fields, the arguments in 2 imply that if $\phi \in \mathbb{C}_{K}(z)$ is defined over a locally compact subfield of $\mathbb{C}_{K}$, and if the Julia set of $\phi$ contains no critical points, then $\phi$ has no wandering domains.

We suspect that the condition concerning critical points is unnecessary. However, the condition that $\phi$ be defined over a locally compact field is crucial. Indeed, the main result of this paper is the following theorem. It shows that if the residue field $\hat{k}$ has positive characteristic, then there are many polynomials defined over the (not locally compact) field $\mathbb{C}_{K}$ which have wandering domains in $\mathbb{C}_{K}[z]$.

Theorem. Let $\mathbb{C}_{K}$ be an algebraically closed complete non-archimedean field with residue field $\hat{k}$ and residue characteristic char $\hat{k}=p>0$. Let $a_{0} \in \mathbb{C}_{K}$ with $\left|a_{0}\right|>1$, and let $\varepsilon>0$. There is some $a \in \mathbb{C}_{K}$ with $\left|a-a_{0}\right| \leq \varepsilon$ such that the Fatou set $\mathcal{F}$ of the function

$$
\phi_{a}(z)=(1-a) z^{p+1}+a z^{p}
$$

has a wandering component.

A weaker version of the above result appeared in [5], with some details of the proof omitted. There, it was assumed that $\mathbb{C}_{K}=\mathbb{C}_{p}$, and it was only shown that there is at least one $a \in \mathbb{C}_{p}$ giving a wandering domain. Indeed, an examination of that proof reveals that the point $a$ constructed has $|a|=|p|^{-(p-1)}$. Moreover, the proof of the main theorem of [5] fails for fields $\mathbb{C}_{K}$ of positive characteristic.

Although the statement and proof of the above Theorem is similar in structure to its predecessor in [5], it is much stronger. First, the Lemmas in Section 4 of the current paper are more complicated than their analogues in [5], in order to apply to the more general class of fields $\mathbb{C}_{K}$ (including fields of characteristic $p$, not just residue characteristic $p$ ). Second, a slight modification of the main proof allows the broader statement that the set of parameters $a$ giving wandering domains is dense in the large open subset $\left\{a \in \mathbb{C}_{K}:|a|>1\right\}$ of the parameter space. That large subset cannot be extended; there can be no wandering domains for $\phi_{a}$ with $|a| \leq 1$, because such $\phi_{a}$ are conjugate to maps of the form $\psi(z)=z^{p+1}+c z^{p}$ with $|c| \leq 1$, which have good reduction and therefore empty Julia set.

Our proof uses some computations specific to this family, but the general method should apply to a larger class of functions. In particular, the main properties of $\phi_{a}$ required in the proof are that it maps a disk $U_{0}$ (in this case, about 0) onto itself with degree divisible by $p$, and another disk $U_{1}$ (in this case, about 1 ) onto $U_{0}$ with degree prime to $p$. In the case that $\mathbb{C}_{K}=\mathbb{C}_{p}$ has characteristic zero, Fernández [10] has extended the argument in [5] to show that there is an open set in the higher-dimensional parameter space of all degree $(p+1)$ polynomials in $\mathbb{C}_{p}[z]$ for which a dense subset of parameters exhibit wandering domains. (She adds our map $\phi_{a}$ to an arbitrary polynomial $Q$ with coefficients of sufficiently small absolute value and shows, by essentially the same argument as in [5], that $a$ can be adjusted by an arbitrarily small amount to guarantee that $Q+\phi_{a}$ has a wandering domain.) It should be straightforward to reproduce Fernández' argument for the more general case of $\mathbb{C}_{K}[z]$ using the methods of this paper, where we do not assume, as Fernández and [5] do, that one may divide by $p$. However, the proof of our Theorem is already so heavy with notation that we prefer to avoid the further tedium of proving the higher-dimensional density statement. Still, we hope that the interested reader will be able to glean enough 
from our proof and from that of Fernández to deduce how the statement can be extended to the higher-dimensional parameter space.

Some comments are in order regarding the field of definition of the parameters $a$ in the Theorem. As previously mentioned, the results of 2] imply that if $a$ were defined over a locally compact subfield $K \subseteq \mathbb{C}_{K}$, and if $\phi_{a}$ had no critical points in its Julia set (that is, if $\phi_{a}$ were hyperbolic), then $\phi_{a}$ would have no wandering domains. However, the parameter $a$ chosen in our proof cannot lie in a discretely valued field. Indeed, the resulting map $\phi_{a}$ will, after many iterations, map all points $z$ in the wandering disk $U_{0}$ to points $\phi_{a}^{n}(z)$ for which the ratio $\log |z| / \log \left|\phi_{a}^{n}(z)\right|$ of valuations is a rational number of arbitrarily large denominator. Such a situation could not occur if the map $\phi_{a}$ were defined over a discretely valued field; in particular, $a$ cannot lie in any locally compact subfield of $\mathbb{C}_{K}$. In other words, the truth of the Theorem above requires the freedom to choose $a \in \mathbb{C}_{K}$; a condition like $a \in \overline{\mathbb{Q}}_{p}$ would be too restrictive.

\section{NON-ARCHIMEDEAN DISKS, MAPPING LEMMAS, AND DYNAMICS}

We will denote the closed disk of radius $r>0$ about a point $a \in \mathbb{C}_{K}$ by $\bar{D}_{r}(a)$, and the open disk by $D_{r}(a)$. We recall some basic properties of non-archimedean disks. Every disk is both open and closed as a topological set. Any point in a disk $U$ is a center, but the radius of $U$ is a well-defined real number $\operatorname{rad}(U)$, which is the same as the diameter of $U$. If two disks in $\mathbb{C}_{K}$ intersect, then one contains the other.

The following lemma describes the action of polynomials on disks. It is easy to prove using basic non-archimedean analysis results, such as Hensel's Lemma and the Weierstrass Preparation Theorem. (See Lemmas 2.2 and 2.6 of [6. Section 2]; see also [18, Chapter 6] for a broad introduction to such techniques over $p$-adic fields.)

Lemma 1.1. Let $U \subseteq \mathbb{C}_{K}$ be a disk with $\operatorname{rad}(U)=r$, and let $f \in \mathbb{C}_{K}[z]$ be a non-constant polynomial. Then:

a. $f(U)$ is a disk.

b. For any $x, y \in U$,

$$
|f(x)-f(y)| \leq \frac{s}{r} \cdot|x-y|
$$

where $s=\operatorname{rad}(f(U))$.

c. $f: U \rightarrow f(U)$ is bijective if and only if inequality (11) attains equality for every $x, y \in U$.

The projective line $\mathbb{P}^{1}\left(\mathbb{C}_{K}\right)$ is equipped with a natural spherical metric

$$
\Delta(x, y)=\frac{|x-y|}{(\max \{|x|, 1\})(\max \{|y|, 1\})}
$$

analogous to the spherical metric on $\mathbb{P}^{1}(\mathbb{C})$. It is easy to verify that $\Delta(x, y)=|x-y|$ if $|x|,|y| \leq 1$; that $\Delta(x, y)=\left|x^{-1}-y^{-1}\right|$ if $|x|,|y| \geq 1$; and that $\Delta(x, y)=1$ otherwise.

Let $\phi \in \mathbb{C}_{K}(z)$ be a rational function, and consider the dynamical system of the iterates $\left\{\phi^{n}\right\}$ acting on $\mathbb{P}^{1}\left(\mathbb{C}_{K}\right)$. As in complex dynamics, we define the Fatou set $\mathcal{F}$ of $\phi$ to the the set of all points $x \in \mathbb{P}^{1}\left(\mathbb{C}_{K}\right)$ such that $\left\{\phi^{n}: n \geq 0\right\}$ is equicontinuous at $x$ with respect to $\Delta$; we define the Julia set $\mathcal{J}$ to be the complement $\mathbb{P}^{1}\left(\mathbb{C}_{K}\right) \backslash \mathcal{F}$.

Following any of [4, 7, 17, it is possible to partition the Fatou set of $\phi$ into "components" using a different definition than the usual topological connected components. (The various 
definitions differ slightly, but they agree on the question of whether or not wandering domains exist for a given map.) Rather than repeating the theoretical definitions, we refer to Theorem 5.4 of [4], which provides the following characterization of Fatou components in our situation.

Specifically, suppose that $\phi$ is a polynomial of degree at least two and the Julia set of $\phi$ is nonempty; let $\mathcal{F}$ denote the Fatou set of $\phi$. If $x \in \mathcal{F}$ has bounded forward orbit (that is, there exists $R>0$ such that $\left|\phi^{n}(x)\right|<R$ for all $n \geq 0$ ), then the component $V$ of $\mathcal{F}$ containing $x$ is the largest closed disk $\bar{D}_{r}(x)$ containing $x$ and contained in $\mathcal{F}$. (At least, that is the case for any of the definitions appearing in [4. For the definition in [17] and the remaining definitions in [7, the component is the open disk $D_{r}(x)$ of the same radius.) Moreover, for such $x$, the component of $\phi^{n}(x)$ is precisely $\phi^{n}(V)$.

We recall Hsia's criterion [12] for equicontinuity, which is a non-archimedean analogue of the Montel-Carathéodory Theorem.

Lemma 1.2. (Hsia) Let $F \subseteq \mathbb{C}_{K}[z]$ be a family of polynomials, and let $U \subseteq \mathbb{C}_{K}$ be a disk. Suppose that there is a point $y \in \mathbb{C}_{K}$ such that for all $f \in F$ and $x \in U$, we have $f(x) \neq y$. Then $F$ is an equicontinuous family.

Hsia stated his result for arbitrary non-archimedean meromorphic functions; in that setting, the criterion is that there are two points in $\mathbb{P}^{1}\left(\mathbb{C}_{K}\right)$ which are omitted by every $f \in F$. In our simpler rephrasing above, the first point is $y$, and the second is $\infty$. Lemma 1.2 follows easily from Lemma 1.1 by first making a change of coordinates to move $y$ to 0 . Any $f(U)$ is a disk not containing 0 (by Lemma 1.1. a and the hypotheses); therefore it either does not intersect $\bar{D}_{1}(0)$ or is contained in $\bar{D}_{1}(0)$. If the former, then $f(U)$ has radius at most 1. If the latter, then the image of $f(U)$ under $1 / z$ is a disk of radius at most 1 . Because $z \mapsto 1 / z$ preserves the spherical metric, equicontinuity follows from Lemma 1.1,b.

\section{The FAMiLY}

Recall that $p=$ char $\hat{k}>0$. For any $a \in \mathbb{C}_{K}$, we define

$$
\phi_{a}(z)=(1-a) z^{p+1}+a z^{p}
$$

as in the Theorem. Note that 0 and 1 are fixed points of $\phi_{a}$. The point at 0 is superattracting (meaning that $\phi_{a}^{\prime}(0)=0$, and therefore nearby points are strongly attracted to 0 under iteration), and if $|a|>1$, then the point at 1 is repelling (meaning that $\left|\phi_{a}^{\prime}(1)\right|>1$, and therefore nearby points are pushed away from 1 under iteration).

The following lemma gives a fairly accurate estimate for the expansion or contraction of distances under a single application of $\phi_{a}$.

Lemma 2.1. Let $a \in \mathbb{C}_{K}$ with $|a|>1$, and let $y_{1}, y_{2} \in \mathbb{C}_{K}$ with $\left|y_{1}\right| \geq\left|y_{2}\right|$. Then

$$
\left|\phi_{a}\left(y_{1}\right)-\phi_{a}\left(y_{2}\right)\right| \leq\left|y_{1}-y_{2}\right| \cdot|a| \cdot\left|y_{1}\right|^{p-1} \cdot \max \left\{|p|,\left|y_{1}\right|,\left(\frac{\left|y_{1}-y_{2}\right|}{\left|y_{1}\right|}\right)^{p-1}\right\} \text {. }
$$

Furthermore, if $y_{1}, y_{2} \in D_{1}(1)$, then $\left|\phi_{a}\left(y_{1}\right)-\phi_{a}\left(y_{2}\right)\right|=|a| \cdot\left|y_{1}-y_{2}\right|$.

Proof of Lemma 2.1. We have

$$
\phi_{a}\left(y_{1}\right)-\phi_{a}\left(y_{2}\right)=(1-a)\left(y_{1}^{p+1}-y_{2}^{p+1}\right)+a\left(y_{1}^{p}-y_{2}^{p}\right)
$$




$$
=a\left(y_{1}-y_{2}\right) \cdot\left[\left(\frac{1}{a}-1\right)\left(\sum_{j=0}^{p} y_{1}^{j} y_{2}^{p-j}\right)+\left(y_{1}-y_{2}\right)^{p-1}+p\left(\sum_{j=0}^{p-1} A_{j} y_{1}^{j} y_{2}^{p-1-j}\right)\right],
$$

with $A_{j} \in \mathbb{Z}$. Thus,

$$
\left|\phi_{a}\left(y_{1}\right)-\phi_{a}\left(y_{2}\right)\right| \leq\left|y_{1}-y_{2}\right| \cdot|a| \cdot \max \left\{\left|y_{1}\right|^{p},\left|y_{1}-y_{2}\right|^{p-1},|p| \cdot\left|y_{1}\right|^{p-1}\right\},
$$

as claimed. Finally, if $y_{1}, y_{2} \in D_{1}(1)$, then

$$
\sum_{j=0}^{p} y_{1}^{j} y_{2}^{p-j} \in D_{1}(1) \quad \text { and } \quad\left|y_{1}-y_{2}\right|<1
$$

In that case, then, (2) has absolute value $|a| \cdot\left|y_{1}-y_{2}\right|$.

\section{Mapping Properties of the Iterates}

In this section, we will apply Lemma 2.1 to describe the behavior of iterates $\phi_{a}^{i}$ near the fixed points at $z=0,1$, for $i$ relatively small.

Fix $a_{0} \in \mathbb{C}_{K}$ with $\left|a_{0}\right|>1$ (as chosen in the hypotheses of the Theorem), and to simplify future notation, define

$$
R=\left|a_{0}\right|^{-1 /(p-1)}, \quad \mu=\max \{|p|, R\}, \quad \text { and } \quad S=\mu R^{3} .
$$

Note that $0<S<R^{-2} S<R \leq \mu<1$. The quantity $R$ represents the radius of a particular disk about 0. Specifically, for $a \in \mathbb{C}_{K}$ with $|a|=\left|a_{0}\right|>1$,

$$
\phi_{a}\left(\bar{D}_{R}(0)\right)=\bar{D}_{R}(0) ;
$$

the mapping is $p$-to-1. The smaller quantity $S$ is important because $\phi_{a}^{i}$ acts in a predictable manner on disks of radius at most $S$ just outside $\bar{D}_{R}(0)$, as shown in the following lemma.

Lemma 3.1. Let $a \in \mathbb{C}_{K}$ with $|a|=\left|a_{0}\right|$, and let $m \geq 1$. Let $x \in \mathbb{C}_{K}$ with $R<|x| \leq$ $R^{1-p^{-m}}$. Then for all $0 \leq i \leq m$, we have

$$
\left|\phi_{a}^{i}(x)\right|=R^{1-p^{i}}|x|^{p^{i}} \leq 1, \quad \text { and } \quad \phi_{a}^{i}\left(\bar{D}_{S}(x)\right) \subseteq \bar{D}_{S_{i}^{\prime}}\left(\phi_{a}^{i}(x)\right),
$$

where

$$
S_{i}^{\prime}=\mu^{i} R^{-e_{i}} S<\mu^{i} R^{-2} S, \quad \text { and } \quad e_{i}=p^{1-m}+p^{2-m}+\cdots+p^{i-m} .
$$

Proof of Lemma 3.1. Observe that $e_{i}$ is a partial sum of a geometric series, so that $e_{i}<p /(p-1) \leq 2$. In particular, the inequality $S_{i}^{\prime}<\mu^{i} R^{-2} S$ is valid. Meanwhile, for any $y \in D_{1}(0),\left|\phi_{a}(y)\right|=|a| \cdot|y|^{p}$, and so the first statement follows from a simple induction.

We now prove the second statement of the lemma by induction on $i$. If $i=0$, in which case $e_{i}=0$, the statement is clear. Given that the statement holds for some $0 \leq i \leq m-1$, write $y=\phi_{a}^{i}(x)$ to simplify notation. Note that $S_{i}^{\prime}=\mu^{i+1} R^{3-e_{i}}<\mu R$, and that

$$
\frac{(\mu R)^{p-1}}{|y|^{p}}<\frac{\mu}{|y|} \leq \max \left\{\frac{|p|}{|y|}, 1\right\} \text {. }
$$

Therefore, by Lemma 2.1.

$$
\phi_{a}\left(\bar{D}_{S_{i}^{\prime}}(y)\right) \subseteq \bar{D}_{\lambda(y) \cdot S_{i}^{\prime}}\left(\phi_{a}(y)\right), \quad \text { where } \quad \lambda(y)=|a| \cdot|y|^{p} \max \left\{1, \frac{|p|}{|y|}\right\} .
$$


We compute

$$
\begin{aligned}
\lambda(y) & =\lambda\left(\phi_{a}^{i}(x)\right)=|a| \cdot\left|\phi_{a}^{i}(x)\right|^{p} \cdot \max \left\{1,\left|\frac{p}{\phi_{a}^{i}(x)}\right|\right\} \\
& \leq|a|\left(R^{1-p^{i}}|x|^{p^{i}}\right)^{p} \cdot \max \left\{1, \frac{|p|}{R}\right\} \\
& =|a| R^{p-1-p^{i+1}}|x|^{p^{i+1}} \mu=\mu R^{-p^{i+1}}|x|^{p^{i+1}} \leq \mu R^{-p^{i+1-m}} .
\end{aligned}
$$

Thus, $\lambda(y) \cdot S_{i}^{\prime} \leq S_{i+1}^{\prime}$. By the inductive hypothesis, $\phi_{a}^{i}\left(\bar{D}_{S}(x)\right) \subseteq \bar{D}_{S_{i}^{\prime}}(y)$; hence, by (44),

$$
\phi_{a}^{i+1}\left(\bar{D}_{S}(x)\right) \subseteq \bar{D}_{S_{i+1}^{\prime}}\left(\phi_{a}(y)\right),
$$

and the Lemma follows.

Remark 3.2. a. The upper bound of $S_{i}^{\prime}$ for the image radius in Lemma 3.1 is far from sharp. The sharp bound is not difficult to compute, but our Theorem will require only a bound which decreases to 0 as $i$ increases; the simpler bound given above will suffice.

$b$. Intuitively, Lemma 3.1 says the following. A point $x$ just outside of $\bar{D}_{R}(0)$ is pushed further away from 0 by $\phi_{a}$. However, $\left|\phi_{a}^{\prime}(x)\right|<1$, so that locally (i.e., on $\bar{D}_{S}(x)$ ), $\phi_{a}$ is contracting. The situation is illustrated in Figure 1, where the small disk $\bar{D}_{r_{1}}\left(x_{1}\right)$ maps to the smaller disk $\bar{D}_{r_{2}}\left(x_{2}\right)$ and then to the even smaller disk $\bar{D}_{r_{3}}\left(x_{3}\right)$. However, at the same time, with $s_{i}=\left|x_{i}\right|$, the disk $\bar{D}_{s_{1}}(0)$ maps to the larger disk $\bar{D}_{s_{2}}(0)$ and then to the even larger disk $\bar{D}_{s_{3}}(0)$. In broad terms, $\phi_{a}$ is locally contracting on the whole annulus

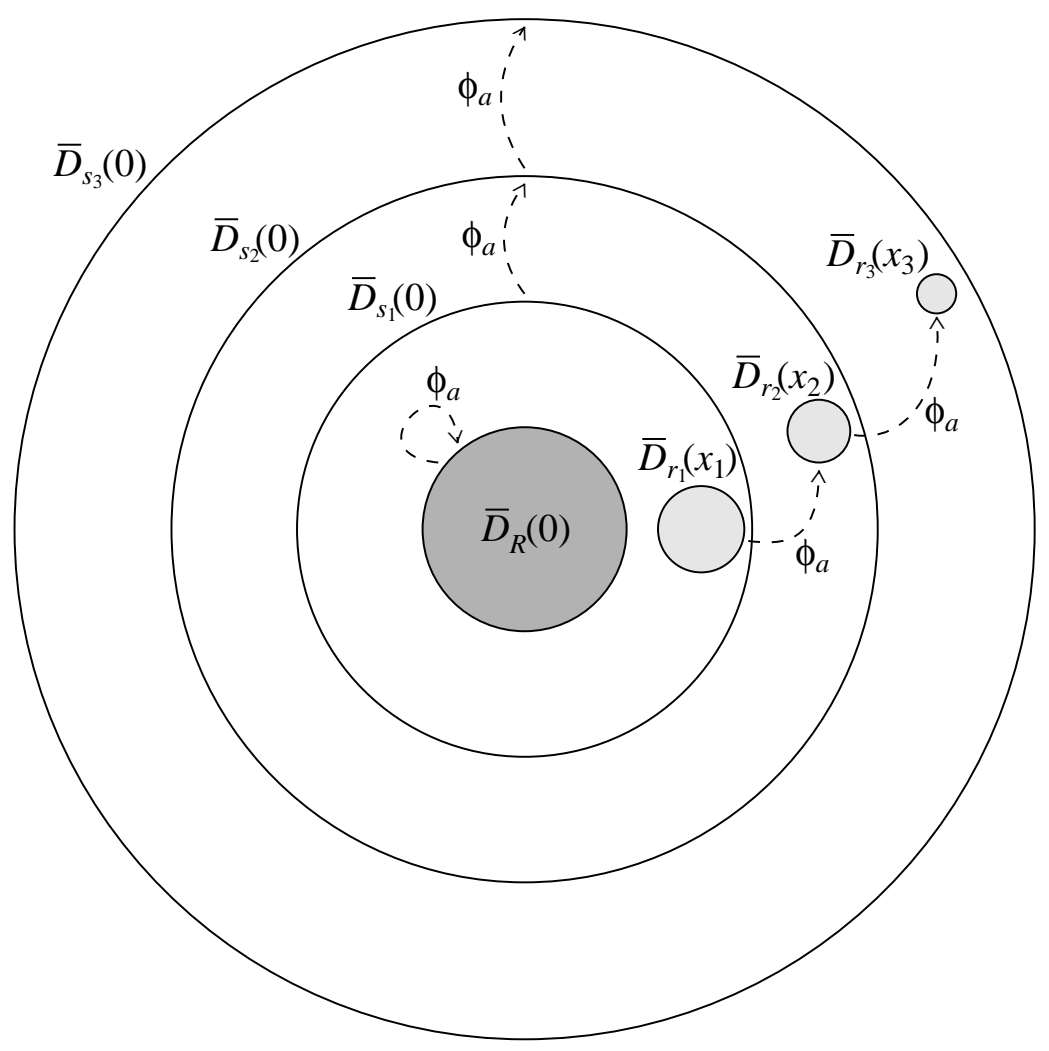

Figure 1. Global expansion and local contraction under $\phi_{a}$. 
$R<|x|<1$, even as every point in the annulus is pushed away from 0 . Thus, if $|x|$ is only slightly bigger than $R$, the iterates of $x$ will stay inside the larger disk $D_{1}(0)$ for some time; and the corresponding iterates of $\bar{D}_{S}(x)$ will become very small in radius.

c. Ultimately, our main interest in Lemma 3.1 lies in the case that $i=m$. However, the cases $i<m$ are important both for the inductive proof and because we need to have some idea of the orbit traversed by $x$ during those $m$ iterations. An analogous remark applies to the quantity $M$ in Lemma 3.3 below.

Lemma 3.3. Let $a \in \mathbb{C}_{K}$ with $|a|=\left|a_{0}\right|$, and let $M \geq 1$. Let $x \in \mathbb{C}_{K}$ with $|x-1| \leq|a|^{-M}$. Then for all $0 \leq i \leq M$, we have

$$
\left|\phi_{a}^{i}(x)-1\right|=|a|^{i} \cdot|x-1|
$$

and for any $0<r \leq|a|^{-M}$,

$$
\phi_{a}^{i}\left(\bar{D}_{r}(x)\right)=\bar{D}_{|a|{ }^{i} r}\left(\phi_{a}^{i}(x)\right) .
$$

Proof of Lemma 3.3. The result follows easily by induction on $i$ using the first statement of Lemma 2.1 and the fact that $\phi_{a}(1)=1$.

\section{VARYing THE PARAMETER}

We wish to study the effects of perturbations of $a$ on the dynamics of $\phi_{a}$, and so we introduce the following notation. For any $z \in \mathbb{P}^{1}\left(\mathbb{C}_{K}\right), a \in \mathbb{C}_{K}$, and $n \geq 0$, define

$$
\Phi_{n}(a, z)=\phi_{a}^{n}(z)
$$

For any fixed $x \in \mathbb{C}_{K}, \Phi_{n}(a, x)$ is a polynomial in the variable $a$. In particular, for any disk $D \in \mathbb{C}_{K}$, the set $\Phi_{n}(D, x)$ is also a disk, by Lemma 1.1.a.

Lemma 4.1. Let $a \in \mathbb{C}_{K}$ with $|a|=\left|a_{0}\right|$, let $M \geq 0$, let $n \geq 1$, and let $x \in \mathbb{C}_{K}$ with $\left|\phi_{a}^{n}(x)-1\right| \leq|a|^{-M}$. Let $\varepsilon \in\left(0,|a|^{1-M}\right]$. Suppose that $\Phi_{n}(\cdot, x)$ maps $\bar{D}_{\varepsilon}($ a) bijectively onto $\bar{D}_{\varepsilon /|a|}\left(\phi_{a}^{n}(x)\right)$. Then $\Phi_{n+M}(\cdot, x)$ maps $\bar{D}_{\varepsilon}(a)$ bijectively onto $\bar{D}_{\varepsilon \cdot|a|(M-1)}\left(\phi_{a}^{n+M}(x)\right)$.

Proof. We proceed by induction on $M$; the case $M=0$ is vacuously true. For $M \geq 1$, let $b_{1}, b_{2} \in \bar{D}_{\varepsilon}(a)$, let $y_{1}=\Phi_{n+M-1}\left(b_{1}, x\right)$, and let $y_{2}=\Phi_{n+M-1}\left(b_{2}, x\right)$. Note that

$$
\begin{aligned}
\left|y_{1}-1\right| & =|a|^{M-1} \cdot\left|\Phi_{n}\left(b_{1}, x\right)-1\right| \\
& \leq|a|^{M-1} \cdot \max \left\{\left|\Phi_{n}\left(b_{1}, x\right)-\phi_{a}^{n}(x)\right|,\left|\phi_{a}^{n}(x)-1\right|\right\} \\
& \leq|a|^{M-1} \cdot \max \left\{|a|^{-1} \cdot\left|b_{1}-a\right|,|a|^{-M}\right\}=|a|^{-1},
\end{aligned}
$$

where the first equality is by Lemma 3.3. and the rest is by the hypotheses, ultrametricity, and Lemma 1.1.b. Therefore,

$$
\begin{aligned}
\left|\phi_{b_{1}}\left(y_{1}\right)-\phi_{b_{2}}\left(y_{1}\right)\right| & =\left|\left(1-b_{1}\right) y_{1}^{p+1}+b_{1} y_{1}^{p}-\left(1-b_{2}\right) y_{1}^{p+1}-b_{2} y_{1}^{p}\right| \\
& =\left|y_{1}\right|^{p} \cdot\left|y_{1}-1\right| \cdot\left|b_{1}-b_{2}\right| \leq|a|^{-1} \cdot\left|b_{1}-b_{2}\right|,
\end{aligned}
$$

by the above bound and because $\left|y_{1}\right|=1$. On the other hand, by Lemma 2.1 ,

$$
\left|\phi_{b_{2}}\left(y_{1}\right)-\phi_{b_{2}}\left(y_{2}\right)\right|=|a| \cdot\left|y_{1}-y_{2}\right|=|a|^{M-1} \cdot\left|b_{1}-b_{2}\right|
$$

where the final equality is by induction and Lemma 1.1. . In particular, if $b_{1} \neq b_{2}$,

$$
\left|\phi_{b_{1}}\left(y_{1}\right)-\phi_{b_{2}}\left(y_{1}\right)\right|<\left|\phi_{b_{2}}\left(y_{1}\right)-\phi_{b_{2}}\left(y_{2}\right)\right| \text {, }
$$

and therefore

$$
\left|\phi_{b_{1}}\left(y_{1}\right)-\phi_{b_{2}}\left(y_{2}\right)\right|=\left|\phi_{b_{2}}\left(y_{1}\right)-\phi_{b_{2}}\left(y_{2}\right)\right|
$$


by ultrametricity. It follows that

$$
\left|\Phi_{n+M}\left(b_{1}, x\right)-\Phi_{n+M}\left(b_{2}, x\right)\right|=|a|^{M-1} \cdot\left|b_{1}-b_{2}\right| .
$$

The same equality is clear if $b_{1}=b_{2}$; thus, (6) holds for all $b_{1}, b_{2} \in \bar{D}_{\varepsilon}(a)$.

By Lemma 1.1 a, $\Phi_{n+M}\left(\bar{D}_{\varepsilon}(a), x\right)$ is a disk about $\phi_{a}^{n+M}(x)$ of some radius $s$. By (6) and Lemma 1.1. c, $s=\varepsilon \cdot|a|^{(M-1)}$, and the mapping is bijective, as claimed.

Lemma 4.2. Let $a \in \mathbb{C}_{K}$ with $|a|=\left|a_{0}\right|$, let $m \geq 1$, let $n \geq 0$, and let $x \in \mathbb{C}_{K}$ with $\left|\phi_{a}^{n}(x)\right|=R^{1-p^{-m}}$. Let $A>|a|^{-1}$ be a real number, let $\varepsilon \in\left(0, A^{-1} S\right]$, and suppose that

$$
\Phi_{n}\left(\bar{D}_{\varepsilon}(a), x\right) \subseteq \bar{D}_{A \varepsilon}\left(\phi_{a}^{n}(x)\right) \text { and } \mu^{m}<A^{-1} R^{p+1} \text {. }
$$

Then $\Phi_{n+m}(\cdot, x)$ maps $\bar{D}_{\varepsilon}(a)$ bijectively onto $\bar{D}_{\varepsilon /|a|}\left(\phi_{a}^{n+m}(x)\right)$.

Proof. For any $0 \leq i \leq m$, let $e_{i}=p^{1-m}+p^{2-m}+\cdots+p^{i-m}$ as in Lemma 3.1, and let

$$
r_{i}=\max \left\{\mu^{i} R^{-e_{i}} A, R^{p-p^{-(m-i)}}\right\} \text {. }
$$

Note that $r_{i} \leq A$, and therefore $r_{i} \varepsilon \leq A \varepsilon \leq S$. We claim that for any such $i$,

$$
\Phi_{n+i}\left(\bar{D}_{\varepsilon}(a), x\right) \subseteq \bar{D}_{r_{i} \varepsilon}\left(\phi_{a}^{n+i}(x)\right),
$$

with $\Phi_{n+i}(\cdot, x)$ mapping $\bar{D}_{\varepsilon}(a)$ bijectively onto $\bar{D}_{r_{i} \varepsilon}\left(\phi_{a}^{n+i}(x)\right)$ if

$$
i \geq 1 \text { and } \mu^{i} R^{-e_{i}} A<R^{p-p^{-(m-i)}} \text {. }
$$

Condition (8) holds for $i=m$ because $\mu^{m}<A^{-1} R^{p+1}$ by hypothesis and because $e_{m}<2$; moreover, $r_{m}=R^{p-1}=|a|^{-1}$. Thus, to prove the lemma, it suffices to prove the claim.

We proceed by induction on $i$. Since $r_{0}=A$, the $i=0$ case is true by hypothesis. For $1 \leq i \leq m$, pick any two distinct points $b_{1}, b_{2} \in \bar{D}_{\varepsilon}(a)$, and let $y_{1}=\Phi_{n+i-1}\left(b_{1}, x\right)$ and $y_{2}=\Phi_{n+i-1}\left(b_{2}, x\right)$. By Lemma 1.1. b, the inductive hypothesis implies that $\left|y_{1}-y_{2}\right| \leq$ $r_{i-1} \cdot\left|b_{1}-b_{2}\right|$. Note that $\left|y_{1}\right|=\left|y_{2}\right|=R^{1-p^{-(m-i+1)}}$, by Lemma 3.1. Therefore,

$$
\begin{aligned}
\left|\phi_{b_{1}}\left(y_{1}\right)-\phi_{b_{2}}\left(y_{1}\right)\right| & =\left|\left(1-b_{1}\right) y_{1}^{p+1}+b_{1} y_{1}^{p}-\left(1-b_{2}\right) y_{1}^{p+1}-b_{2} y_{1}^{p}\right| \\
& =\left|b_{1}-b_{2}\right| \cdot\left|y_{1}-1\right| \cdot\left|y_{1}\right|^{p}=\left|b_{1}-b_{2}\right| \cdot\left|y_{1}\right|^{p} \\
& =\left|b_{1}-b_{2}\right| \cdot R^{p-p^{-(m-i)}} \leq r_{i}\left|b_{1}-b_{2}\right|,
\end{aligned}
$$

with equality if condition (8) holds. Meanwhile, $\left|y_{1}-y_{2}\right| \leq r_{i-1} \varepsilon \leq S<\mu R$; by Lemma 2.1,

$$
\left|\phi_{b_{2}}\left(y_{1}\right)-\phi_{b_{2}}\left(y_{2}\right)\right| \leq \lambda\left(y_{1}\right) \cdot\left|y_{1}-y_{2}\right| \leq r_{i-1} \cdot \lambda\left(y_{1}\right) \cdot\left|b_{1}-b_{2}\right|
$$

where $\lambda\left(y_{1}\right)=|a| \cdot\left|y_{1}\right|^{p} \max \left\{1,|p| /\left|y_{1}\right|\right\}$ as in the proof of Lemma 3.1. Adding, we have

$$
\left|\Phi_{n+i}\left(b_{1}, x\right)-\Phi_{n+i}\left(b_{2}, x\right)\right|=\left|\phi_{b_{1}}\left(y_{1}\right)-\phi_{b_{2}}\left(y_{2}\right)\right| \leq \max \left\{r_{i}, r_{i-1} \cdot \lambda\left(y_{1}\right)\right\} \cdot\left|b_{1}-b_{2}\right|,
$$

with equality if the maximum is uniquely attained. Thus, to prove (17), it suffices to show

$$
\lambda\left(y_{1}\right) \cdot r_{i-1} \leq r_{i}
$$

By Lemma 1.1, the full claim would follow if in fact $\lambda\left(y_{1}\right) \cdot r_{i-1}<r_{i}$ whenever condition (8) holds.

Note that

$$
\lambda\left(y_{1}\right)=R^{-p^{-(m-i)}} \cdot \max \left\{R,|p| \cdot R^{p^{-(m-i+1)}}\right\} \leq \mu R^{-p^{-(m-i)}} .
$$

Thus, if $r_{i-1}=\mu^{i-1} R^{-e_{i-1}} A$, then

$$
\lambda\left(y_{1}\right) \cdot r_{i-1} \leq \mu^{i} R^{-e_{i}} A \leq r_{i}
$$


with strict inequality if condition (8) holds. Similarly, if $r_{i-1}=R^{p-p^{-(m-i+1)}}$, then

$$
\lambda\left(y_{1}\right) \cdot r_{i-1}=R^{p-p^{-(m-i)}} \cdot \max \left\{R^{1-p^{-(m-i+1)}},|p|\right\}<R^{p-p^{-(m-i)}} \leq r_{i} .
$$

The claim is proved, and the lemma follows.

Remark 4.3. a. The two conditions displayed in the hypotheses of Lemma 4.2 say, first, that $A$ is large enough to measure the size of a certain image disk, and second, that $m$ is large enough that $\mu^{m}$ is smaller than the already small $A^{-1} R^{p+1}$.

$b$. Lemmas 4.1 and 4.2 both describe the behavior of iterates $\phi_{a}^{n+i}(x)$ as a varies; the former is for $\phi_{a}^{n}(x)$ close to 1 , and the latter is for $\phi_{a}^{n}(x)$ just outside $\bar{D}_{R}(0)$. The conclusion of Lemma 4.2 exactly matches the key hypothesis of Lemma 4.1, and the conclusion of Lemma 4.1 implies the key hypothesis of Lemma 4.2. (We shall need the full strength of Lemma 4.1 elsewhere in the main proof.) Thus, we will be able to alternate applications of each Lemma in order to describe the behavior of $\phi_{a}^{n}$ for larger and larger values of $n$.

\section{Proof of the Theorem}

To prove the Theorem, we will first choose several auxiliary sequences $\left\{m_{i}\right\},\left\{M_{i}\right\},\left\{n_{i}\right\}$ and $\left\{N_{i}\right\}$ of positive integers, and $\left\{\varepsilon_{i}\right\}$ and $\left\{r_{i}\right\}$ of positive real numbers. The carefully chosen integer sequences will be used to describe the orbit of a certain point $x$ under various maps $\phi_{a}$; the real values $\varepsilon_{i}$ will be radii of disks in which the parameters $a$ lie; and the $r_{i}$ will be radii of disks in which certain images $\phi_{a}^{n}(x)$ lie.

After those choices, we will argue inductively using Lemmas 4.1 and 4.2 to produce a sequence $\left\{a_{i}\right\}$ of parameters with limit $a$ such that $\phi_{a}$ has a wandering domain.

5.1. Auxiliary sequences. We are given $\varepsilon>0$ and $a_{0} \in \mathbb{C}_{K}$ with $\left|a_{0}\right|>1$. Define $R, S$, and $\mu$ as in (3). We assume without loss that $\varepsilon \leq S$.

Choose a strictly increasing sequence of positive integers $\left\{M_{i}\right\}_{i \geq 1}$ so that

$$
\left|a_{0}\right|^{1-M_{1}} \leq \varepsilon, \quad \text { and for all } i \geq 2, \quad\left|a_{0}\right|^{M_{i-1}-M_{i}} \leq S .
$$

Let $\varepsilon_{0}=\varepsilon$, and for all $i \geq 1$, let

$$
\varepsilon_{i}=\left|a_{0}\right|^{1-M_{i}} S
$$

Then $\left\{\varepsilon_{i}\right\}$ is a strictly decreasing sequence of positive real numbers with limit 0 , and $\left|a_{0}\right|^{1-M_{i}} \leq \varepsilon_{i-1}$ for all $i \geq 1$.

Choose another strictly increasing sequence of positive integers $\left\{m_{i}\right\}_{i \geq 0}$ so that

$$
\mu^{m_{i}} \leq\left|a_{0}\right|^{-M_{i+1}} \cdot R^{2} \quad \text { for all } i \geq 0 .
$$

For all $i \geq 0$, define

$$
r_{i}=R^{1-p^{-m_{i}}}
$$

Then $\left\{r_{i}\right\}$ is a strictly decreasing sequence of positive real numbers with limit $R$. Note that the starting index for $\left\{m_{i}\right\},\left\{\varepsilon_{i}\right\}$, and $\left\{r_{i}\right\}$ is $i=0$, while that for $\left\{M_{i}\right\}$ is $i=1$.

We will find $x \in D_{1}(0)$ and $a \in \bar{D}_{\varepsilon}\left(a_{0}\right)$ so that the orbit $\left\{\phi_{a}^{j}(x)\right\}_{j \geq 0}$ is described by

$$
\underbrace{0, \ldots, 0}_{m_{0}}, \underbrace{1, \ldots, 1}_{M_{1}}, \underbrace{0, \ldots, 0}_{m_{1}}, \underbrace{1, \ldots, 1}_{M_{2}}, \underbrace{0, \ldots, 0}_{m_{2}}, \ldots
$$

where a 0 in the $j$-th position in the sequence indicates that $\phi_{a}^{j-1}(x) \in D_{1}(0)$, and a 1 indicates that $\phi_{a}^{j-1}(x) \in D_{1}(1)$. 
To simplify future notation, define

$$
n_{i}=\sum_{j=1}^{i}\left(m_{j-1}+M_{j}\right), \quad \text { and } \quad N_{i}=n_{i}+m_{i}
$$

for all $i \geq 0$. That is, $n_{i}$ is the number of terms in (9) up to but not including the block of $m_{i} 0$ 's, and $N_{i}$ is the number of terms up to but not including the block of $M_{i+1} 1$ 's.

5.2. Choosing the parameter. Recall that $r_{0}=R^{1-p^{-m_{0}}}$. Pick $y \in \mathbb{C}_{K}$ with $|y|=r_{0}$; by Lemma 3.1. $\left|\phi_{a_{0}}^{m_{0}}(y)\right|=1$. (Such a $y$ exists because $r_{0} \in \mathbb{C}_{K}$.) In addition, $\phi_{a_{0}}^{m_{0}}(0)=0$. Therefore, by Lemma 1.1 a, $\phi_{a_{0}}^{m_{0}}\left(\bar{D}_{r_{0}}(0)\right)$ is a disk containing both 0 and $\phi_{a_{0}}^{m_{0}}(y)$, and hence

$$
\bar{D}_{1}(0) \subseteq \phi_{a_{0}}^{m_{0}}\left(\bar{D}_{r_{0}}(0)\right) .
$$

Thus, there is some $x \in \bar{D}_{r_{0}}(0)$ with $\phi_{a_{0}}^{m_{0}}(x)=1$. Again by Lemma 3.1. we have $|x|=r_{0}$.

For every $i \geq 0$, we will find $a_{i} \in \bar{D}_{\varepsilon_{i-1}}\left(a_{i-1}\right)$ (or just the original $a_{0}$, for $i=0$ ) so that for every $a \in \bar{D}_{\varepsilon_{i}}\left(a_{i}\right)$, the orbit $\left\{\phi_{a}^{j}(x)\right\}$ follows (9) up to the $j=N_{i}$ iterate, with $\phi_{a_{i}}^{N_{i}}(x)=1$ and such that

$$
\Phi_{N_{i}}(\cdot, x): \bar{D}_{\varepsilon_{i}}\left(a_{i}\right) \rightarrow \bar{D}_{\varepsilon_{i} /\left|a_{i}\right|}(1) \quad \text { is bijective. }
$$

Because $\left\{\varepsilon_{i}\right\}$ is decreasing, each $a_{i}$ will lie in $\bar{D}_{\varepsilon_{0}}\left(a_{0}\right)$, and therefore $\left|a_{i}\right|=\left|a_{0}\right|$.

We construct the sequence $\left\{a_{i}\right\}$ inductively. For $i=0$, we already have $\phi_{a_{0}}^{N_{0}}(x)=1$, and by Lemma 3.1] the orbit $\left\{\phi_{a_{0}}^{j}(x)\right\}$ follows (9) up to the $N_{0}=m_{0}$ iterate. By Lemma 4.2 (with $n=n_{0}=0, m=m_{0}, a=a_{0}, A=1$, and $\varepsilon=\varepsilon_{0}$ ), condition (10) holds. Also, by Lemma 3.1. the orbit $\left\{\phi_{a}^{j}(x)\right\}$ is correct up to $j=N_{0}$ for every $a \in \bar{D}_{\varepsilon_{0}}\left(a_{0}\right)$. Hence, the $i=0$ case is already done.

For $i \geq 1$, given $a_{i-1}$ with the desired properties, set $\rho=\left|a_{0}\right|^{1-M_{i}} \leq \varepsilon_{i-1}$. By the inductive hyphothesis, for each $a \in \bar{D}_{\rho}\left(a_{i-1}\right)$, the orbit $\left\{\phi_{a}^{j}(x)\right\}$ agrees with (9) up to $j=$ $N_{i-1}$. Moreover, because $\Phi_{N_{i-1}}(\cdot, x)$ maps $\bar{D}_{\varepsilon_{i-1}}\left(a_{i-1}\right)$ bijectively onto $\bar{D}_{\varepsilon_{i-1} /\left|a_{i-1}\right|}(1)$ with $\Phi_{N_{i-1}}\left(a_{i-1}, x\right)=1$, we see that $\Phi_{N_{i-1}}(\cdot, x)$ also maps $\bar{D}_{\rho}\left(a_{i-1}\right)$ bijectively onto $\bar{D}_{\rho /\left|a_{i-1}\right|}(1)$, by Lemma 1.1.c. By Lemma 4.1 (with $a=a_{i-1}, M=M_{i}, n=N_{i-1}$, and $\varepsilon=\rho$ ), $\Phi_{n_{i}}(\cdot, x$ ) maps $\bar{D}_{\rho}\left(a_{i-1}\right)$ bijectively onto $\bar{D}_{1}(1)$. Hence, there exists $b \in \mathbb{C}_{K}$ such that $\Phi_{n_{i}}(b, x)=0$. By Lemma 1.1]c, we must have $\left|b-a_{i-1}\right|=\rho$; and because $r_{i}=R^{1-p^{-m_{i}}}<1$,

$$
\Phi_{n_{i}}(\cdot, x): \bar{D}_{\sigma}(b) \rightarrow \bar{D}_{r_{i}}(0) \text { is bijective, }
$$

where $\sigma=r_{i} \cdot\left|a_{0}\right|^{1-M_{i}}=r_{i} \cdot \rho \in(0, \rho)$. Moreover, for all $a \in \bar{D}_{\sigma}(b)$,

$$
\left|\Phi_{N_{i-1}}(a, x)-1\right|=\left|a_{0}\right|^{-1}\left|a-a_{i-1}\right|=\left|a_{0}\right|^{-1}\left|b-a_{i-1}\right|=\left|a_{0}\right|^{-M_{i}} .
$$

By Lemma 3.3, then, the orbit $\left\{\phi_{a}^{j}(x)\right\}$ is correct up to $j=n_{i}$ for every $a \in \bar{D}_{\sigma}(b)$.

Choose $c \in \bar{D}_{\sigma}(b)$ so that $\left|\Phi_{n_{i}}(c, x)\right|=r_{i}$. (Such $c$ exists because of (11) and the fact that $r_{i} \in\left|\mathbb{C}_{K}\right|$.) By Lemma 3.1. $\left|\Phi_{N_{i}}(c, x)\right|=1$. Furthermore, it is clear that $\Phi_{N_{i}}(b, x)=0$. Because the polynomial image of a disk is a disk (Lemma 1.1. a), it follows that $\Phi_{N_{i}}\left(\bar{D}_{\sigma}(b, x)\right) \supseteq \bar{D}_{1}(0)$. We may therefore choose $a_{i} \in \bar{D}_{\sigma}(b)$ so that $\Phi_{N_{i}}\left(a_{i}, x\right)=1$.

By Lemma 3.1. we must have $\left|\Phi_{n_{i}}\left(a_{i}, x\right)\right|=r_{i}$. Observe also that $\varepsilon_{i} \leq \sigma$. By (11) and Lemma 1.1. c, $\Phi_{n_{i}}\left(\bar{D}_{\varepsilon_{i}}\left(a_{i}\right), x\right)$ must be a disk of radius $\varepsilon_{i} \cdot\left|a_{0}\right|^{M_{i}-1}=S$. Therefore, by Lemma 4.2 (with $n=n_{i}, m=m_{i}, a=a_{i}, A=\left|a_{i}\right|^{M_{i}-1}$, and $\varepsilon=\varepsilon_{i}$ ), condition (10) holds on $\bar{D}_{\varepsilon_{i}}\left(a_{i}\right)$. By Lemma 3.1] the orbit $\left\{\phi_{a}^{j}(x)\right\}$ is correct up to $j=N_{i}$ for every $a \in \bar{D}_{\varepsilon_{i}}\left(a_{i}\right)$. Our construction of $a_{i}$ is complete. 
The sequence $\left\{a_{i}\right\}_{i \geq 0}$ is a Cauchy sequence, because for any $0 \leq i \leq j$, we have $\left|a_{i}-a_{j}\right| \leq$ $\varepsilon_{i}$, and $\varepsilon_{i} \rightarrow 0$. Therefore, the sequence has a limit $a \in \mathbb{C}_{K}$, with $\left|a-a_{0}\right| \leq \varepsilon_{0}$. We claim that $\phi_{a}$ has a wandering domain.

5.3. The end of the proof. By construction, $a \in \bar{D}_{\varepsilon_{i}}\left(a_{i}\right)$ for every $i \geq 0$; hence, the orbit $\left\{\phi_{a}^{j}(x)\right\}$ follows (9) exactly. In light of Lemmas 3.1 and 3.3, we must have

$$
\left|\phi_{a}^{n_{i}}(x)\right|=r_{i}=R^{1-p^{-m_{i}}}, \quad \text { and } \quad\left|\phi_{a}^{N_{i}}(x)-1\right|=|a|^{-M_{i+1}}
$$

for any $i \geq 0$. Let $U=\bar{D}_{S}(x)$; we will show that $U$ is contained in a wandering domain of the Fatou set $\mathcal{F}$ of $\phi_{a}$.

Every iterate $U_{n}=\phi_{a}^{n}(U)$ is a disk; we claim that for any $i \geq 0$, the radius of $U_{n_{i}}$ is at most $S$, and the radius of $U_{N_{i}}$ is at most $|a|^{-M_{i+1}} S$. The claim is easily proven by induction, as follows. For $i=0, U_{n_{0}}=U_{0}=U$, which has radius $S$; and by Lemma 3.1. $U_{N_{0}}$ has radius at most $\mu^{m_{0}} R^{-2} S \leq|a|^{-M_{1}} S$. For $i \geq 1$, we assume the radius of $U_{N_{i-1}}$ is at most $|a|^{-M_{i}} S$. By Lemma 3.3, the radius of $U_{n_{i}}$ is at most $S$; and by Lemma 3.1, the radius of $U_{N_{i}}$ is at most $\mu^{m_{i}} R^{-2} S \leq|a|^{-M_{i+1}} S$.

If some $U_{n_{i}}$ contained the point 1 , then by Lemma 3.3. some $U_{n_{i+j}}$ would contain $\bar{D}_{1}(0)$ and therefore would have radius greater than $S$. Thus, no $U_{n_{i}}$ contains the point 1 ; and because 1 is fixed, it follows that no $U_{n}$ contains 1. By Hsia's criterion (Lemma 1.2), then, the family $\left\{\phi_{a}^{n}\right\}$ is equicontinuous on $U$, and therefore $U \subseteq \mathcal{F}$.

The iterates of $U$ are bounded away from $\infty \in \mathbb{P}^{1}\left(\mathbb{C}_{K}\right)$, and $\phi_{a}$ is a polynomial. Therefore, by any of the definitions of components in [4, 7, 17], the component $V$ of $\mathcal{F}$ containing $U$ must be a disk [4, Theorem 5.4.d], as must all of its iterates. As before, no iterate $\phi^{n}(V)$ of $V$ can contain the point 1 . In addition, each $\phi^{n}(V)$ is contained either in $D_{1}(0)$ or $D_{1}(1)$, since it is a disk intersecting one of those two disks but not containing 1 . The symbolic dynamics of $V$ are therefore described by equation (9). Because those dynamics are not preperiodic, it follows that $V$ must be wandering.

\section{REFERENCES}

[1] A. Beardon, Iteration of Rational Functions (Springer-Verlag, New York, 1991).

[2] R. Benedetto, ' $p$-adic dynamics and Sullivan's No Wandering Domains Theorem', Compositio Math. 122 (2000), 281-298.

[3] R. Benedetto, 'Hyperbolic maps in p-adic dynamics', Ergodic Theory Dynam. Systems 21 (2001), 1-11.

[4] R. Benedetto, 'Components and periodic points in non-archimedean dynamics', Proc. London Math. Soc. (3) 84 (2002), 231-256.

[5] R. Benedetto, 'Examples of wandering domains in p-adic polynomial dynamics', C. R. Acad. Sci. Paris, Ser. I 335 (2002), 615-620.

[6] R. Benedetto, 'Non-Archimedean holomorphic maps and the Ahlfors Islands theorem', Amer. J. Math. 125 (2003), 581-622.

[7] R. Benedetto, 'Wandering domains and nontrivial reduction in non-archimedean dynamics', Illinois J. Math. 49 (2005), 167-193.

[8] J.-P. Bézivin, 'Sur les points périodiques des applications rationnelles en analyse ultramétrique', Acta Arith. 100 (2001), 63-74.

[9] L. Carleson and T. Gamelin, Complex Dynamics (Springer-Verlag, New York 1991).

[10] G. Fernández, 'Wandering Fatou components on p-adic polynomial dynamics', preprint, 2005; http://arxiv.org/math.DS.0503720. 
[11] M. Herman and J.-C. Yoccoz, 'Generalizations of some theorems of small divisors to non-archimedean fields', Geometric Dynamics (Rio de Janairo, 1981) (ed J. Palis), Lecture Notes in Mathematics 1007 (Springer-Verlag, Berlin-New York 1983), pp. 408447.

[12] L.-C. Hsia, 'Closure of periodic points over a nonarchimedean field', J. London Math. Soc. (2) 62 (2000), 685-700.

[13] N. Koblitz, p-adic Numbers, p-adic Analysis, and Zeta-Functions, 2nd ed. (SpringerVerlag, New York-Berlin, 1984).

[14] J. Lubin, 'Nonarchimedean dynamical systems', Compositio Math. 94 (1994), 321-346.

[15] J. Milnor, Dynamics in One Complex Variable: Introductory Lectures, 2nd ed. (Vieweg, Braunschweig, 2000).

[16] S. Mochizuki, Foundations of p-adic Teichmüller Theory, (Int. Press, Cambridge, MA, 1999).

[17] J. Rivera-Letelier, 'Dynamique des fonctions rationnelles sur des corps locaux', Astérisque 287 (2003), 147-230.

[18] A. Robert, A Course in p-adic Analysis, (Springer-Verlag, New York, 2000).

[19] J.-P. Serre, Corps Locaux, 2nd ed., (Hermann, Paris, 1968).

[20] D. Sullivan, 'Quasiconformal homeomorphisms and dynamics, I, Solution of the FatouJulia problem on wandering domains', Annals of Math. 122 (1985), 401-418.

Department of Mathematics and Computer Science, Amherst College, Amherst, Ma 01002, USA

E-mail address: rlb@cs.amherst.edu

URL: http://www.cs. amherst.edu/ ${ }^{\sim} \mathrm{rlb}$ 